\title{
Management of patients with high-risk and advanced prostate cancer in the Middle East: resource-stratified consensus recommendations
}

\author{
Deborah Mukherji $^{1}$ (1) $\cdot$ Bassem Youssef ${ }^{2} \cdot$ Christelle Dagher $^{1} \cdot$ Albert El-Hajj $^{3} \cdot$ Rami Nasr $^{3} \cdot$ Fadi Geara $^{2}$. \\ Danny Rabah ${ }^{4}$. Saad Al Dousari ${ }^{5} \cdot$ Rabih Said $^{6} \cdot$ Raja Ashou $^{7}$. Wassim Wazzan ${ }^{2}$. Michel Jabbour ${ }^{8} \cdot$ George Farha $^{9}$. \\ Nibras Al Hamdani ${ }^{10}$. Yousuf Al Hallaq ${ }^{10}$. Hassan Ghazal ${ }^{11}$. Haifa Dbouk ${ }^{12}$. Bassel Bachir ${ }^{2}$. Clement El Khoury ${ }^{13}$. \\ Ghazi Sakr ${ }^{14} \cdot$ Hero K. Hussain ${ }^{15} \cdot$ Khaled Sayyid $^{16} \cdot$ Khaled Ibrahim $^{17} \cdot$ Mohammad Haidar $^{15} \cdot$ Nicolas Zouain $^{18}$. \\ Nizar Bitar ${ }^{19}$. Walid Alameh ${ }^{20} \cdot$ Fadi Abbas $^{21}$. Sami Faddoul ${ }^{22}$. Elie Nemer ${ }^{23}$. Georges Assaf ${ }^{8}$. Fadi Farhat ${ }^{17}$. \\ Muhammad Bulbul $^{2} \cdot$ Sally Temraz ${ }^{1}$ - Ali Shamseddine ${ }^{1} \cdot$ Silke Gillessen $^{24,25} \cdot$ Aurelius Omlin $^{25} \cdot$ Raja Khauli $^{3}$
}

Received: 21 May 2019 / Accepted: 4 July 2019 / Published online: 11 July 2019

(c) The Author(s) 2019

\begin{abstract}
Purpose Prostate cancer care in the Middle East is highly variable and access to specialist multidisciplinary management is limited. Academic tertiary referral centers offer cutting-edge diagnosis and treatment; however, in many parts of the region, patients are managed by non-specialists with limited resources. Due to many factors including lack of awareness and lack of prostate-specific antigen (PSA) screening, a high percentage of men present with locally advanced and metastatic prostate cancer at diagnosis. The aim of these recommendations is to assist clinicians in managing patients with different levels of access to diagnostic and treatment modalities.

Methods The first Advanced Prostate Cancer Consensus Conference (APCCC) satellite meeting for the Middle East was held in Beirut, Lebanon, November 2017. During this meeting a consortium of urologists, medical oncologists, radiation oncologist and imaging specialists practicing in Lebanon, Syria, Iraq, Kuwait and Saudi Arabia voted on a selection of consensus questions. An additional workshop to formulate resource-stratified consensus recommendations was held in March 2019.

Results Variations in practice based on available resources have been proposed to form resource-stratified recommendations for imaging at diagnosis, initial management of localized prostate cancer requiring therapy, treatment of castration-sensitive/ naïve advanced prostate cancer and treatment of castration-resistant prostate cancer.

Conclusion This is the first regional consensus on prostate cancer management from the Middle East. The following recommendations will be useful to urologists and oncologists practicing in all areas with limited access to specialist multidisciplinary teams, diagnostic modalities and treatment resources.
\end{abstract}

Keywords Prostate cancer $\cdot$ Middle East $\cdot$ Resource-stratified recommendations $\cdot$ Consensus $\cdot$ Multidisciplinary

Electronic supplementary material The online version of this article (https://doi.org/10.1007/s00345-019-02872-x) contains supplementary material, which is available to authorized users.

Deborah Mukherji

Dm25@aub.edu.lb

Raja Khauli

rkhauli@aub.edu.lb

Extended author information available on the last page of the article

\section{Introduction}

The 2017 Advanced Prostate Cancer Consensus Conference (APCCC) was held in St Gallen, Switzerland in March 2017 during which a panel of 60 international experts voted on 150 questions addressing controversial topics in prostate cancer management [1]. The first APCCC Satellite Meeting for the Middle East was held in Beirut, Lebanon in November 2017 in conjunction with the Middle East Prostate Cancer Consortium (MEPCC) comprised of urologists, medical oncologists, radiation oncologists and imaging specialists largely from Lebanon, Syria and Iraq with expert urologists from Kuwait and Saudi Arabia joined by the co-chair of 
Fig. 1 Areas of consensus ( $\geq 75 \%$ agreement) APCCC 2017 compared to Beirut Satellite Meeting 2017
Lymph node dissection in men with $\mathrm{cN} 0 \mathrm{cM} 0$ high-risk prostate cancer undergoing prostatectomy

\begin{tabular}{|l|l|l|}
\hline APCCC 2017 & Beirut Satellite 2017 & Concordance? \\
\hline $84 \%$ & $76 \%$ & Yes \\
\hline
\end{tabular}

In men post-prostatectomy without lymph node involvement on surgical pathology (pNO), with undetectable postoperative PSA and who have recovered urinary continence, do you recommend adjuvant radiation therapy in in case of:

$\underline{\text { Positive surgical margins }}$

- Yes, in the majority of patients

\begin{tabular}{|l|l|l|}
\hline APCCC 2017 & Beirut Satellite 2017 & Concordance? \\
\hline $45 \%$ & $81 \%$ & No \\
\hline
\end{tabular}

If you recommend adjuvant radiation therapy in men with pN1 disease (adequate sampling) post-prostatectomy, what field of radiation therapy do you recommend in the majority of men?

- Prostatic bed plus whole pelvis

\begin{tabular}{|l|l|l|}
\hline APCCC 2017 & Beirut Satellite 2017 & Concordance? \\
\hline $97 \%$ & $82 \%$ & Yes \\
\hline
\end{tabular}

Do you recommend adding ADT in combination with salvage radiation therapy?

- Yes, in the majority of patients

\begin{tabular}{|l|l|l|}
\hline APCCC 2017 & Beirut Satellite 2017 & Concordance? \\
\hline $61 \%$ & $83 \%$ & No \\
\hline
\end{tabular}

\section{Oligometastatic + Bone Health}

\section{Definition of oligometastatic disease}

A clinically meaningful definition of oligometastatic prostate cancer that influences treatment decision (local treatment of all lesions +/- systemic therapy) includes:

- Only patients with a limited number of bone and/or lymph nodes metastases that can be treated with local therapy

\begin{tabular}{|l|l|l|}
\hline APCCC 2017 & Beirut Satellite 2017 & Concordance? \\
\hline $61 \%$ & $81 \%$ & No \\
\hline
\end{tabular}

Do you recommend a dental check for CRPC patients with bone metastases prior to starting an osteoclast-targeted therapy?

- Yes, in the majority of patients

\begin{tabular}{|l|l|l|}
\hline APCCC 2017 & Beirut Satellite 2017 & Concordance? \\
\hline $74.8 \%$ & $82 \%$ & Yes \\
\hline
\end{tabular}

the APCCC Dr Aurelius Omlin from St Gallen. During this satellite meeting, faculty members presented brief clinical updates with a focus on topics of particular relevance to the Middle East region and all attendees were asked to vote on a selection of the APCCC questions (see supplemental material). Consensus was declared if $\geq 75 \%$ of participants 
Fig. 1 (continued)

\section{Castration-sensitive/naive metastatic prostate cancer}

For men who are suitable for chemotherapy:

Do you recommend Docetaxel in addition to ADT in men with de novo metastatic castration-sensitive/naive prostate cancer and high volume disease as defined by CHAARTED (visceral metastases and/or $\geq 4$ bone lesions with $\geq 1$ beyond vertebral bodies and pelvis)?

- Yes, in the majority of patients

\begin{tabular}{|l|l|l|}
\hline APCCC 2017 & Beirut Satellite 2017 & Concordance? \\
\hline $96 \%$ & $95 \%$ & Yes \\
\hline
\end{tabular}

Do you recommend Docetaxel in addition to ADT in with metastatic castrationsensitive/naive disease relapsing after prior treatment for localized prostate cancer and with high volume disease as per CHAARTED (visceral metastases and/or $\geq 4$ bone lesions with $\geq 1$ beyond vertebral bodies and pelvis)?

- Yes, in the majority of patients

\begin{tabular}{|l|l|l|}
\hline APCCC 2017 & Beirut Satellite 2017 & Concordance? \\
\hline $74 \%$ & $89 \%$ & No \\
\hline
\end{tabular}

\section{Castration-Resistant Prostate Cancer}

What is your preferred first-line MCRPC treatment option in the majority of asymptomatic or minimally symptomatic men who did NOT receive Docetaxel in the castrationsensitive/naive setting?

- Abiraterone or Enzalutamide

\begin{tabular}{|l|l|l|}
\hline APCCC 2017 & Beirut Satellite 2017 & Concordance? \\
\hline $86 \%$ & $87 \%$ & Yes \\
\hline
\end{tabular}

What is your preferred first-line MCRPC treatment option in the majority of symptomatic men who DID receive Docetaxel in the castration-sensitive/naive setting?

- Abiraterone or Enzalutamide

\begin{tabular}{|l|l|l|}
\hline APCCC 2017 & Beirut Satellite 2017 & Concordance? \\
\hline $90 \%$ & $86 \%$ & Yes \\
\hline
\end{tabular}

What is your preferred second-line MCRPC treatment option in the majority of men with symptomatic $\mathrm{MCRPC}$ who had progressive disease as best response to first-line Abiraterone or Enzalutamide?

- Taxane

\begin{tabular}{|l|l|l|}
\hline APCCC 2017 & Beirut Satellite 2017 & Concordance? \\
\hline $96 \%$ & $85 \%$ & Yes \\
\hline
\end{tabular}

who did not vote for "unqualified" or "abstain" and chose the same option [2] (see Fig. 1). The meeting was organized by the Continuing Medical Education office of the American University of Beirut and co-chaired by Dr Deborah Mukherji (medical oncologist) and Dr Raja Khauli (urologist and panel member for the APCCC 2017 meeting).

The manuscript published following the APCCC 2017 provides a guide for clinicians to assist in the discussions 
Fig. 1 (continued)

What is your preferred second-line MCRPC treatment option in the majority of men with symptomatic $\mathrm{MCRPC}$ and secondary (acquired) resistance (initial response followed by progression) after use of first-line Abiraterone or Enzalutamide?

- Taxane

\begin{tabular}{|l|l|l|}
\hline APCCC 2017 & Beirut Satellite 2017 & Concordance? \\
\hline $90 \%$ & $100 \%$ & Yes \\
\hline
\end{tabular}

What is your preferred third-line MCRPC treatment option in the majority of men with MCRPC, progressing on or after second-line Docetaxel for mCRPC AND prior treatment with Abiraterone or Enzalutamide?

- Cabazitaxel

\begin{tabular}{|l|l|l|}
\hline APCCC 2017 & Beirut Satellite 2017 & Concordance? \\
\hline $61 \%$ & $81 \%$ & No \\
\hline
\end{tabular}

with patients as part of a multidisciplinary decision-making process particularly in areas lacking clear evidence from randomized clinical trials on which to base treatment recommendations [1]. Due to the rapid changes in prostate cancer management since this publication of the APCCC recommendations in 2017, an additional workshop was held in Beirut in March 2019 to update our resource-stratified consensus recommendations specifically for the Middle East using a modified-Delphi method. Resource-stratified recommendations are based on expert-opinion and structured in-line with a simplified version of the resource-stratification levels proposed by the Breast Health Global Initiative (BHGI) and adopted by the American Society of Clinical Oncology (ASCO) [3]. We have stratified our recommendations between two levels rather than four since the choice of treatment in the Middle East region is not just dependent on financial resources but also available expertise. We did not feel that for the needs of practitioners in the region, making finer discrimination between the four levels would be of added value since there would be considerable overlap and repetition. We emphasize the importance of referral to centers with appropriate expertise as needed wherever possible. Significant variations in coverage of health-care costs exist both within and between countries. Systems of governmental health-care coverage, private insurance and self-payment operate in parallel. In a region lacking access to specialist multidisciplinary care in many areas, these recommendations are not designed to replace evidence-based guidelines, however, may assist in management decisions for patients with different levels of access to diagnostic and treatment modalities.

\section{Epidemiology of prostate cancer in the Middle East}

Prostate cancer is the second most commonly diagnosed cancer in men worldwide, with an incidence of $1,276,106$ in 2018 and the 5th most common cause of cancer mortality worldwide with 358,989 fatalities in 2018, according to Globocan [4]. Australia, Europe and Northern America have the highest age-standardized incidence rate of prostate cancer in 2018, when Asia has the lowest incidence rate [4].

A dramatic increase in prostate cancer incidence has been globally identified in the past few decades since the introduction of PSA screening [5]. In 2018, prostate cancer has been identified as the most common cancer in men in all the continents except for Asia where it is the fifth most common cancer (Table 1) [4]. Nevertheless, it is worth noting that the reported incidence in the past 5 years in the US has dropped since the United States Preventative Services Task Force (USPSTF) issued a recommendation

Table 1 Incidence of Prostate cancer in 2018 worldwide

\begin{tabular}{lll}
\hline Area & $\begin{array}{l}\text { Incidence of prostate } \\
\text { cancer in 2018 }\end{array}$ & $\begin{array}{l}\text { Age-standardized } \\
\text { ratio per 100,000 }\end{array}$ \\
\hline Europe & 449,761 & 62.1 \\
Asia & 297,215 & 11.5 \\
North America & 234,278 & 73.7 \\
Latin America and the & 190,385 & 56.4 \\
$\quad$ Caribbean & & \\
Africa & 80,971 & 26.6 \\
Oceania & 23,496 & 79.1
\end{tabular}


Table 2 Incidence of prostate cancer in the Middle East region, 2018

\begin{tabular}{lcc}
\hline Middle East country & $\begin{array}{l}\text { Incidence of prostate } \\
\text { cancer in 2018 }\end{array}$ & $\begin{array}{l}\text { Age-standardized } \\
\text { ratio per 100,000 }\end{array}$ \\
\hline Lebanon & 1503 & 39.3 \\
Iraq & 556 & 6.6 \\
Jordan & 397 & 14.7 \\
Kuwait & 221 & 21.6 \\
Oman & 145 & 12.7 \\
Qatar & 73 & 15.5 \\
Saudi Arabia & 607 & 6.1 \\
Syria & 1136 & 20.1 \\
Algeria & 2578 & 13.0 \\
Egypt & 3109 & 9.5 \\
Libya & 317 & 15.6 \\
Morocco & 3990 & 22.7 \\
Tunisia & 819 & 12.3 \\
\hline
\end{tabular}

against screening in 2012, with concomitant rise in locally advanced disease and nodal metastasis at diagnosis [6].

Partly due to the lack of awareness and regular screening in the Middle East, plus potential deficiencies in regional cancer registries, the incidence of prostate cancer is low compared to the rest of the world. Lebanon, a country with advanced tertiary care referral centers, has the highest rate of prostate cancer in the region due to high use of PSA screening with 39.3 per 100,000 in most recent data in 2018 (Table 2).

\section{Diagnosis and management of high-risk localized and locally advanced prostate cancer}

In common with many parts of the world, cancer care in the Middle East is highly variable with specialist multidisciplinary care limited to few academic medical centers in the region. PSA screening is not routinely practiced, and our panelists reported that a significant proportion of men with prostate cancer present with locally advanced and metastatic disease. Few prospective data are available, however, a single-institution study from the American University of Beirut, Lebanon showed that up to $25 \%$ of patients presented with locally advanced or metastatic disease including $16 \%$ with M1 disease [7].

\section{Imaging at diagnosis}

Recent data from the PRECISION and PROMIS studies have established the value of performing magnetic resonance imaging (MRI) prior to prostate biopsy in men with suspected prostate cancer, however, our panel discussed the fact that few radiologists in the region have specific training in reading prostate MRI according to the prostate imaging reporting and data system (PIRADS) classification [8, 9]. For patients who have access to MRI pre-biopsy, targeted and random biopsies from the prostate are recommended, either "cognitive" targeting or image-guided according to the expertise of the treating team. For patients without access to MRI pre-biopsy, trans-rectal ultrasound (TRUS)-guided biopsy remains standard of care. If TRUS-guided biopsy is not available, patients should be referred to centers offering this modality; finger-directed biopsy of the prostate should only be performed in patients with grossly advanced local disease. For patients with high-risk disease at diagnosis, the panel considered that the current standard of care remains bone scan and cross-sectional imaging with either computed tomography (CT) or MRI. However, for patients with access to positron emission tomography (PET)-PSMA or wholebody MRI scans (so-called 'next-generation imaging'), these imaging modalities are increasingly being utilized for staging, particularly PET-PSMA in Lebanon and Saudi Arabia (Table 3). To date there is no evidence that the use of next-generation imaging for staging improves outcomes. The panel discussed the controversy surrounding the management of patients found to have distant metastatic disease on next-generation imaging since treatment guidelines are based on patients staged with conventional modalities. We recommend that patients diagnosed with stage 4 disease on next-generation imaging should not be offered radical

Table 3 Resource-stratified recommendations for imaging at diagnosis of prostate cancer

\begin{tabular}{llll}
\hline Resource-level & $\begin{array}{l}\text { Imaging prior to } \\
\text { biopsy }\end{array}$ & Biopsy & Imaging to rule-out metastatic disease in high-risk patients \\
\hline Basic/limited & None & $\begin{array}{c}\text { TRUS-guided biopsy or finger } \\
\text { directed in grossly advanced } \\
\text { local disease }\end{array}$ & Bone scan plus or minus CT or MRI \\
Enhanced/maximal & MRI & $\begin{array}{l}\text { Targeted plus random } \\
\text { Cognitive or fusion-image } \\
\text { guided if local expertise and } \\
\text { access }\end{array}$ & $\begin{array}{l}\text { Bone scan plus CT } \\
\text { PET-PSA if accessible } \\
\end{array}$ \\
& & Whole-body MRI if accessible and local expertise \\
\hline
\end{tabular}


prostatectomy outside clinical trials, however, given recent data from the STAMPEDE trial, radiation to the primary in low-volume metastatic disease is now standard of care and should be offered [10].

\section{Initial management of localized/locally advanced disease}

The definition of high-risk localized and locally advanced prostate cancer varies between guidelines and inclusion criteria for key clinical trials such as the STAMPEDE study [1]. For the APCCC 2017 St Gallen meeting, the European Association of Urology (EAU) guideline definition was used (high-risk localized disease: PSA level $>20 \mathrm{ng} / \mathrm{ml}$, or Gleason score $>7$ or $>\mathrm{T} 2 \mathrm{c}$; locally advanced disease: any PSA level, any Gleason score, cT3-4, or cN+).

In regions with limited access to primary care physicians, the majority of men with prostate cancer present to a general urologist. Due to lack of formal multidisciplinary tumor board meetings outside academic medical centers, the urologist who makes the diagnosis of prostate cancer is usually responsible for setting the treatment plan and making referrals to other specialists. Our panel recommends that ideally all men with locally advanced prostate cancer should be discussed with and seen by a radiation oncologist and a medical oncologist for formulation of the management plan (Table 4). Physicians with no access to an on-site tumor board can present cases virtually or in person at institutions where multidisciplinary meetings are being held. Other options include direct telephone/email contact between local urologists, medical oncologists and radiation oncologists. Multidisciplinary discussion is essential at diagnosis but also important at relapse following radical local therapy.

The St Gallen APCCC 2017 conference did not address the choice of primary treatment for high-risk localized and locally advanced prostate cancer. In the Middle East region since many patients are diagnosed with prostate cancer by general urologists, our panelists noted that in some cases men with locally advanced or metastatic disease at diagnosis are offered androgen-deprivation therapy (ADT) alone and not referred for radiation or additional systemic therapy despite new data suggesting a survival benefit to treatment intensification for this group of patients [11-14]. Our panelists also discussed that at the other end of the spectrum, patients presenting with locally advanced disease may be offered surgery as part of a multimodality treatment; patients have to be informed about the high possibility of requiring adjuvant radiation therapy. Our group is working on the development of patient information in regional languages describing different treatment modalities and their potential toxicities.

\section{Surgical management of high-risk localized and locally advanced prostate cancer plus or minus adjuvant radiation therapy}

Our panel discussed the fact that younger patients without medical co-morbidities are increasingly opting for primary surgery; however, education about the possible need for adjuvant radiation therapy should be discussed with all patients, ideally with referral to a radiation oncologist (Table 4). Our panelists agreed with the APCCC 2017 consensus regarding pelvic lymph node (PLND) dissection for the majority of men with high-risk prostate cancer undergoing radical prostatectomy (Fig. 1). Extended PLND dissection to the level of the common iliac arteries was also a consensus recommendation, however, our panel qualified that this should only be undertaken by surgeons with appropriate training and expertise.

For centers with appropriate resources and expertise, open radical prostatectomy and robotic-assisted laparoscopic prostatectomy are options to discuss with patients. Ideally,

Table 4 Resource-stratified recommendations for initial management of localized prostate cancer requiring therapy

\begin{tabular}{|c|c|c|c|}
\hline Resource-level & Multidisciplinary discussion & $\begin{array}{l}\text { Surgical management of localized } \\
\text { prostate cancer }\end{array}$ & Radiation for localized prostate cancer \\
\hline Basic/limited & $\begin{array}{l}\text { Review of published guidelines } \\
\text { Discussion of options with patient by } \\
\text { primary physician } \\
\text { Telephone/email discussion between } \\
\text { urologist/oncologist/radiation oncolo- } \\
\text { gist } \\
\text { Patient referral to other specialists for } \\
\text { management }\end{array}$ & $\begin{array}{l}\text { Open radical prostatectomy } \pm \text { pelvic } \\
\text { lymph node dissection (for non-low } \\
\text { risk patients) }\end{array}$ & $\begin{array}{l}\text { External-beam radiation therapy with } \\
\text { addition of ADT for intermediate- } \\
\text { high risk disease }\end{array}$ \\
\hline Enhanced/maximal & $\begin{array}{l}\text { Face-to-face tumor board with imaging } \\
\text { and pathology review } \\
\text { Patient referral to urologist, oncologist } \\
\text { and radiation oncologist to discuss } \\
\text { options for management }\end{array}$ & $\begin{array}{l}\text { Consider radical prostatectomy (open or } \\
\text { robotic-assisted laparoscopic depend- } \\
\text { ing on local expertise) } \\
\text { Extended lymph node dissection with } \\
\text { for men with high-risk disease }\end{array}$ & $\begin{array}{l}\text { Consider IMRT with ADT for inter- } \\
\text { mediate/high-risk disease } \pm \text { moder- } \\
\text { ate hypofractionation } \\
\text { Consider brachytherapy if available } \\
\text { and appropriate }\end{array}$ \\
\hline
\end{tabular}


patients with high-risk and locally advanced disease requesting surgery should be referred to high-volume centers. Our panel stressed the importance of patient education regarding rigorous follow-up post-prostatectomy using PSA monitoring to ensure that patients who will require salvage radiation therapy will be identified at an appropriately early time period with low PSA levels (see salvage radiation section).

\section{Adjuvant radiation after radical prostatectomy}

Patients post-prostatectomy with an undetectable postoperative PSA but high-risk pathologic features should be considered for adjuvant radiation therapy; however, the decision between offering adjuvant radiation versus early salvage radiation is controversial and an overall survival benefit associated with pure adjuvant treatment has not been demonstrated [15]. The results of the on-going RADICALS (NCT00541047) and RAVES (NCT00860652) studies which have been designed to compare adjuvant and early savage radiation therapy may inform future practice.

Our panel reached consensus that the majority of highrisk patients with positive surgical margins should be offered adjuvant radiation therapy. The importance of giving adjuvant therapy based on positive margins alone has been debated in the literature; however, recent data suggest that the risk of recurrence and even prostate cancer-specific mortality may be higher in patients with positive margins and high-risk disease [16]. For patients with seminal vesicle involvement or Gleason grade group 5 tumors, or even certain patients with node-positive tumors but no other highrisk features and undetectable post-operative PSA, the option of adjuvant versus early salvage radiation can be discussed. Adjuvant treatment for node-negative disease is generally given to the prostate bed and pelvis with ADT for 6 months [17]; however, consensus was not reached in our panel on this issue, as results from the NRG/RTOG 0534 trial are still not fully published. Our panel reached consensus that adjuvant radiation for men with node-positive disease should be given to the prostate bed plus whole pelvis with at least 18-36 months of ADT.

The results of randomized studies comparing purely adjuvant versus salvage radiation therapy are awaited, however, in terms of cost-effectiveness in low-resource settings, if appropriate PSA monitoring can be undertaken our panel recommends deferring radiation to the early salvage setting in men with evidence of rising PSA post-prostatectomy.

\section{Salvage radiation after radical prostatectomy}

Men who fail to achieve an undetectable PSA $(<0.2 \mathrm{ng} / \mathrm{ml})$ post-prostatectomy [18] and those with rising PSA after an initial drop to undetectable levels are candidates for salvage radiation. Current data suggest that starting early salvage radiation therapy before the PSA rises to 0.5 will have better outcomes [19, 20]. Our panel reflected that many patients do not have appropriate follow-up of post-prostatectomy and present for salvage with much higher PSA levels. On the other extreme, patients may opt to measure their PSA post-operatively at very frequent intervals and present with evidence of rising PSA at lower levels than the standard $0.2 \mathrm{ng} / \mathrm{ml}$ definition of biochemical recurrence. The panel considered that recommended salvage radiation in men with a confirmed rising PSA prior to the $0.2 \mathrm{ng} / \mathrm{ml}$ threshold is appropriate (two successive rises of PSA) [21].

The panel discussed that if resources are available, a PET-PSMA can be useful in the context of patients presenting post-prostatectomy with PSA $>0.5 \mathrm{ngml}$ to evaluate for nodal or distant metastatic disease [22] and tailor the need and extent of radiation salvage.

A consensus was reached regarding the recommendation of ADT (LHRH-Agonist preferred over anti-androgen) with salvage radiation therapy; however, there was no consensus over the duration and in general 6-12 months are recommended according to the commonly used regimens in published trials $[17,23]$.

\section{Systemic therapy for node-positive disease post-prostatectomy}

The management of patients with high-risk, locally advanced prostate cancer found to have positive lymph nodes post-radical prostatectomy is a controversial issue. Recent data from the STAMPEDE study and NRG Oncology/RTOG 0521 study $[11,12,24]$ were discussed at our update workshop held in March 2019. These studies provide data to support the use of docetaxel for six cycles or abiraterone for 2 years with radiation and ADT for 2 years in selected patients. Our panel reached consensus that ideally men found to have node-positive disease post-prostatectomy should see both a radiation oncologist and a medical oncologist for discussion regarding radiation plus systemic therapy (Table 5).
Table 5 High-risk features to consider adjuvant systemic therapy (docetaxel/abiraterone)
STAMPEDE criteria

NRG Oncology/RTOG 0521 study criteria
At least 2 of: $\mathrm{T} 3$ or $4, \mathrm{PSA} \geq 40 \mathrm{ng} / \mathrm{ml}$, Gleason 8-10 Stage $\mathrm{pT}_{\text {any }} \mathrm{pN}+\mathrm{M} 0$

Gleason 9-10 independent of PSA or T stage Gleason 7-8 and PSA $\geq 20 \mathrm{ng} / \mathrm{ml}$ with any T stage

Gleason score 8 and PSA $<20 \mathrm{ng} / \mathrm{ml}$ with T stage $\geq \mathrm{T} 2$ 


\section{Definitive radiation therapy for high-risk and locally advanced prostate cancer}

For patients undergoing definitive radiation therapy for high-risk and locally advanced prostate cancer, techniques vary depending on local resources and expertise (Table 4). For men with access to enhanced or maximal resources in the region, several centers are now offering Intensity Modulated Radiation Therapy (IMRT) which has benefits in terms of minimizing toxicity related to treatment [25]. Recent ASTRO/ASCO/AUA guidelines recommend moderate hypofractionation across risk groups, however, the task force strongly recommended image-guided radiation therapy and avoidance of non-modulated three-dimensional conformal techniques with any hypofractionated approach [26].

For patients with high-risk disease receiving definitive radiation therapy, 36 months of ADT was not shown to be superior to 18 months of ADT. In this randomized study, overall survival was not significantly different between the two groups; however, non-inferiority could not be established [27]. Our panel currently recommend at least 18 months ADT for the majority of high-risk patients, noting that improvements in imaging and the early identification of patients with metastatic disease is likely to refine patient selection.

Investigators from The American University of Beirut Medical Center (AUBMC) have shown that nadir PSA at $0.06 \mathrm{ng} / \mathrm{ml}$ is a strong predictor of outcome in a cohort of patients with intermediate and high-risk localized prostate cancer [28].

\section{Management of advanced castration-sensitive/naïve prostate cancer}

For patients with biochemical recurrence after radical prostatectomy and salvage radiation or primary radiation therapy, evidence-based recommendations for the timing of initiation of ADT have not been well defined. Recent clinical trials have shown a benefit in terms of prolongation of metastasisfree survival from the addition of enzalutamide, apalutamide or darolutamide in non-metastatic castration-resistant prostate cancer (nmCRPC) [29-31]. As yet, these medications are not widely available in the Middle East for this indication, however, may be considered proof of principle that earlier control of androgen receptor (AR) signaling in patients with a low burden of disease in the advanced setting may improve outcomes. Regarding the timing of initiation of ADT in men after failure of local therapy, consensus was not reached, however, $34 \%$ of panel recommend starting ADT in the majority of patients with confirmed PSA progression and $58 \%$ recommended starting ADT in a minority of selected patients for example PSA $\geq 4 \mathrm{ng} / \mathrm{ml}$ with doubling time less than 6 months. Only 8\% recommend starting ADT only on detection of metastatic disease.

Our panel discussed the fact that recent trials in the setting of nmCRPC used conventional imaging for staging and that with the wider used of PET-PSMA we are seeing fewer patients with rising PSA and no radiologic evidence of metastatic disease. We discussed the limited sensitivity of PET$\mathrm{CT}$ at PSA levels less than $0.5 \mathrm{ng} / \mathrm{ml}$ [22].

\section{Oligometastatic disease}

As previously discussed, a high proportion of patients in the Middle East present with advanced prostate cancer at diagnosis. This may be due to various reasons including lack of screening, awareness and health-care access. The management of so-called "oligometastatic" prostate cancer is a controversial topic discussion at most international meetings and the subject of on-going prospective research. Our panel discussed the fact that since many patients in the region lack access to uro-oncology specialists or clinical trials, this is an area that is being addressed largely at specialist centers after multidisciplinary discussion. With the increasing use of PET-PSMA scans at diagnosis particularly in Lebanon, we are seeing more patients who may be candidates for intensification of both local and systemic therapy.

The APCCC 2017 panel did not reach consensus on the definition of oligometastatic disease with $10 \%$ of the experts stating that they did not believe that oligometastatic disease exists as a clinically meaningful entity. All of our panel in Beirut considered oligometastatic disease to be a clinically meaningful entity. There was consensus that patients with a limited number of bone and/or lymph nodes who could be treated with local therapy would constitute a clinically meaningful definition of oligometastatic prostate cancer that may influence treatment decisions (local treatment of all lesions \pm systemic therapy). Where available, our panel recommends either PET-PSMA or whole-body MRI scan (depending on local expertise) for the confirmation of oligometastatic disease.

For the management of patients diagnosed with de novo oligometastatic disease with no prior treatment for prostate cancer, in the absence of clinical trials, $50 \%$ of our panelists voted for radical treatment of lesions including the primary with ADT for 24-26 months plus or minus systemic treatment with docetaxel or abiraterone, however, consensus was not reached (Table 4).

\section{Metastatic castration-sensitive/naïve prostate cancer (not considered oligometastatic)}

For men progressing following local therapy or presenting with de novo metastatic disease, data from the CHAARTED, STAMPEDE and LATTITUDE studies have conclusively 
demonstrated a survival benefit from the addition of systemic therapy to standard ADT [11-14]. It is clear from the data that patients with a higher disease burden derive the greatest benefit from additional systemic therapy; however, the definition of high-volume disease has not been consistent across trials. Our panel considered that a practical definition of high-volume metastatic disease for multidisciplinary decision making is the definition used in the CHAARTED study of visceral metastasis or $\geq 4$ bone lesions with $\geq 1$ beyond the vertebral bodies and pelvis with the caveat that any imaging modality can be used.

We reached consensus that for men suitable for chemotherapy, docetaxel or abiraterone in addition to ADT should be recommended for castration-sensitive/naïve patients with high-volume metastatic disease as defined above (either de novo at diagnosis or at relapse following local therapy).

The cost-effectiveness of abiraterone plus prednisone until progression versus six cycles of docetaxel was discussed. No formal head-to-head comparisons have been made, however, the authors of the STAMPEDE study have published an indirect comparison between patients who were randomized to either the ADT plus docetaxel arm of the trial and the ADT plus abiraterone arm of the trial during the same time period. The investigators report no difference in overall survival between the two groups [32]. Despite the fact that generic forms of abiraterone will soon be available in the region, six cycles of docetaxel are currently significantly more cost-effective compared to abiraterone plus prednisone until progression [33]. The panel considered this to be the treatment of choice for patients who are candidates for chemotherapy.

For patients with low-volume disease as defined by the CHAARTED study, the choice of additional systemic therapy is more controversial. Long-term survival analysis of the CHAARTED study confirmed a significant survival benefit associated with docetaxel for patients with highvolume disease, however, no survival benefit was observed for patients with low-volume disease. In view of these data, many consider the evidence base to be stronger for the use of abiraterone for low-volume hormone-sensitive metastatic disease $[11,13,34]$. In view of the associated cost and limited access to this treatment in most of the region, our panel recommend that treatment with docetaxel should be considered, particularly for patients with poor adverse prognostic factors such as high Gleason grade group [32] (Table 6).

Recently published data from the STAMPEDE study have also clarified the role of radiation to the prostate in patients with advanced disease. Local radiation did not improve survival for unselected patients; however, a prespecified analysis showed that survival was improved (from 73 to $81 \%$ at 3 years) in those with a low metastatic burden [10].

Our panel reached consensus that baseline imaging and follow-up imaging at PSA nadir/completion of six cycles of docetaxel should be performed with further imaging at progression defined by confirmed PSA rise or clinical progression. Next-generation imaging was recommended if available, however, the standard of care remains $\mathrm{CT}$ and bone scans.

\section{Management of castration-resistant prostate cancer}

Access to treatment modalities for metastatic castration-resistant prostate cancer (mCRPC) in the region varies according to country and financial coverage. In Lebanon, the ministry of public health covers abiraterone or enzalutamide (but not

Table 6 Resource-stratified recommendations for the treatment of castration-sensitive/naïve advanced prostate cancer

\begin{tabular}{|c|c|c|c|}
\hline Resource-level & Oligometastatic & $\begin{array}{l}\text { Low-volume metastatic disease (not consid- } \\
\text { ered oligometastatic) }\end{array}$ & High-volume metastatic disease \\
\hline Basic/limited & $\begin{array}{l}\text { ADT_-surgical/medical } \\
\text { Consider radiation to } \\
\text { prostate if local treat- } \\
\text { ment has not been } \\
\text { given }\end{array}$ & $\begin{array}{l}\text { ADT_-surgical/medical } \\
\text { Consider radiation to prostate if local treat- } \\
\text { ment has not been given }\end{array}$ & $\begin{array}{l}\text { ADT_-surgical/medical, consider docetaxel } 6 \\
\text { cycles }\end{array}$ \\
\hline Enhanced/maximal & $\begin{array}{l}\text { Consider PET-PSMA/ } \\
\text { whole-body MRI } \\
\text { Radiation to prostate if } \\
\text { local treatment has not } \\
\text { been given } \\
\text { Consider radia- } \\
\text { tion to metastatic } \\
\text { lesions + ADT mini- } \\
\text { mum 24-36 months } \\
\text { Consider abiraterone } \\
2 \text { years with radiation } \\
\text { or docetaxel } 6 \text { cycles }\end{array}$ & $\begin{array}{l}\text { Radiation to prostate if local treatment has not } \\
\text { been given } \\
\text { ADT-lifelong (surgical/medical) } \\
\text { Consider abiraterone until progression (if } \\
\text { available) or docetaxel } 6 \text { cycles }\end{array}$ & $\begin{array}{l}\text { No local therapy indicated unless for palliation } \\
\text { ADT-lifelong (surgical/medical) } \\
\text { Consider docetaxel } 6 \text { cycles (preferred in terms } \\
\text { of cost-effectiveness) or abiraterone until } \\
\text { progression if available }\end{array}$ \\
\hline
\end{tabular}


sequential therapy), docetaxel and cabazitaxel for eligible patients. We are not aware of any data suggesting excess toxicity or frequent need for dose modifications for men in the region compared to published data. Sipuleucel-T and radium-223 are not available in the region. Lutetium-PSMA therapy is available in Lebanon, however, since this is not FDA or EMEA approved it is not covered by third-party payers.

A randomized phase II study has reported non-inferiority in terms of PSA metrics between low-dose abiraterone $250 \mathrm{mg}$ with a low-fat meal compared to standard dosing with $1000 \mathrm{mg}$ fasting in patients CRPC [35]. Additional studies are required to assess the long-term efficacy of the strategy, however in resource-limited environments this could potentially be considered.

Our panel agreed with the APCCC 2017 consensus that asymptomatic men with $\mathrm{mCRPC}$ should receive abiraterone or enzalutamide as first-line treatment whether they had received ADT alone (87\%) or ADT plus docetaxel in the castration-naïve setting ( $86 \%$ ). This was qualified by the recommendation that in men with progression less than 6 months following the completion of 6 cycles of docetaxel in the castration-naïve setting, cabazitaxel could be considered. The panel also discussed the need to consider biopsy in patients progressing with visceral disease, particularly with a low PSA who may be developing androgen-independent disease and in some cases neuro-endocrine differentiation [36].

For asymptomatic men with mCRPC and progression on abiraterone or enzalutamide, the panel preferred taxane chemotherapy as a second-line option (56\%), however, consensus was not reached.

We reached consensus that for symptomatic patients with acquired resistance to first-line abiraterone/enzalutamide, taxane chemotherapy should be offered (100\%), and that cabazitaxel should be offered as third-line treatment for the majority of men with mCRPC progressing following second-line docetaxel and prior treatment with abiraterone/ enzalutamide (81\%). The panel discussed the possible use of docetaxel re-challenge in selected patients with mCRPC when cabazitaxel is not available (Table 7).
Our panel preferred baseline imaging and follow-up imaging at PSA nadir and again at progression, however, consensus was not reached and some opted for baseline imaging only and monitoring by PSA alone with imaging at progression. Next-generation imaging was preferred if available, otherwise $\mathrm{CT}$ and bone scan remain standard imaging modalities. Our panel reflected that in many cases, lines of therapy are switched based on PSA progression alone without imaging; however, ideally patient should be monitored by cross-sectional imaging.

\section{Use of osteoclast-targeted therapy}

Our panel reflected that the monitoring of bone health for patients with prostate cancer treated with ADT in the region is sub-optimal. A useful resource is the FRAX online calculator (https://www.sheffield.ac.uk/FRAX/) that is freely available and has been validated in several middle-eastern populations [37]. All patients on ADT should be prescribed calcium and vitamin D supplementation with monitoring for osteoporosis.

The RANK-ligand inhibitor denosumab and bisphosphonate zoledronic acid are both available in the region; however, denosumab is only approved for the treatment of osteoporosis and not advanced cancer with bone metastasis in some countries. Neither drug has been shown to improve survival or influence progression-free survival, however, in the setting of mCRPC both drugs can protect against skeletal-related events (SREs) [38-40]. There is no evidence to support the use of osteoclast-targeted therapy in the nonmetastatic setting or metastatic castration-naïve patients for SRE prevention (Table 8).

Our panel reached consensus that osteoclast-targeted therapy for SRE prevention should be recommend in the majority of patient with $\mathrm{mCRPC}$ and bone metastasis. A dental checkup should be performed prior to starting therapy to decrease the risk of osteonecrosis of the jaw. Most of the panel recommend zoledronic acid for 2 years which can be given every 3 months and may be more cost-effective that denosumab that should be given every 4 weeks continuously [41].

Table 7 Resource-stratified recommendations for the treatment of castration-resistant prostate cancer

\begin{tabular}{|c|c|c|c|c|}
\hline Resource-level & Asymptomatic mCRPC & Symptomatic mCRPC & Second-line mCRPC & $\begin{array}{l}\text { Third-line } \\
\text { mCRPC }\end{array}$ \\
\hline Basic/limited & Docetaxel & Docetaxel & $\begin{array}{l}\text { Docetaxel re-challenge in } \\
\text { selected patients } \\
\text { Supportive care }\end{array}$ & Supportive care \\
\hline Enhanced/maximal & $\begin{array}{l}\text { Abiraterone/enzalutamide } \\
\text { Docetaxel }\end{array}$ & $\begin{array}{l}\text { Docetaxel } \\
\text { Abiraterone/enzalutamide }\end{array}$ & $\begin{array}{l}\text { Docetaxel/cabazitaxel } \\
\text { Abiraterone/Enzalutamide } \\
\text { Consider biopsy-if low } \\
\text { PSA/visceral disease }\end{array}$ & $\begin{array}{l}\text { Cabazitaxel } \\
\text { Consider biopsy-if } \\
\text { low PSA/visceral } \\
\text { disease } \\
\text { Consider PSMA-based } \\
\text { theranostics if avail- } \\
\text { able }\end{array}$ \\
\hline
\end{tabular}


Table 8 Resource-stratified recommendations for monitoring bone health and the use of osteoclast-targeted therapy

\begin{tabular}{llll}
\hline Resource-level & Monitoring bone heath on ADT & $\begin{array}{l}\text { Non-osteoporotic patients with } \\
\text { localized/advanced HSPC }\end{array}$ & $\begin{array}{l}\text { Non-osteoporotic patients with mCRPC (normal renal } \\
\text { function) }\end{array}$ \\
\hline $\begin{array}{l}\text { Basic/limited } \\
\text { Enhanced/maximal }\end{array}$ & $\begin{array}{l}\text { DXA scan after 2 years on ADT } \\
\text { DXA scan at start of ADT and } \\
\text { every 2 years on treatment }\end{array}$ & $\begin{array}{l}\text { No osteoclast-directed therapy } \\
\text { No osteoclast-directed therapy }\end{array}$ & $\begin{array}{l}\text { Consider zoledronic acid } 4 \text { mg IV every } 3 \text { months } \\
\text { Consider zoledronic acid } 4 \text { mg IV every 4-12 weeks } \\
\text { Consider denosumab 120 mg s/c every 4 weeks }\end{array}$ \\
\hline
\end{tabular}

\section{Conclusion}

This satellite meeting of the APCCC-MEPCC is the first regional consensus on prostate cancer management in the Middle East that has attempted to set out recommendations based on availability of local resources and expertise. Our group acknowledges that participants were largely from Lebanon and from tertiary referral centers in the region. This reflects our management approach, in particular our use of novel diagnostic imaging modalities such as PET-PSMA which is widely available in Lebanon. We hope this review of controversial issues in prostate cancer management will be useful to the non-specialist urologist or oncologist practicing in all areas with limited access to specialist multidisciplinary teams, diagnostic and treatment modalities.

Acknowledgements Dr. Bob Djavan, Dr. Maurice Haddad, Dr. Sleiman Merhej, Dr. Toufic Eid, Dr. Youssef Zeidan, Dr. Maroun Moukarzel, Dr. Georges Nassar, Dr. Selim Zeineh, Dr. Hazem Assi, Dr. Nadim Ayoub, Dr. Abdel Razak Nweir, Dr. Ahmad Al Mohammed, Dr. Al Hadi Al Seoudi, Dr Ali Abdulameer, Dr. Ali Wafa Zaki, Dr. Antoine Abi Raad, Dr. Bahaulddin Al Kamolei, Dr. Fadi Saadeh, Dr. Fadi Merhi, Dr. Firas Al Jorani, Dr. Hussein Sabbagh, Dr. Mounes Abo Mansour, Dr. Mustafa Al Suwaychit, Dr. Nada Abedalla, Dr. Namir Dallouji, Dr. Nawras Jaafar, Dr. Nidal Kheder, Dr. Saad Al Sammarraie, Dr. Usama Al Nasiri, Dr. Violette Dib, Dr. Yaala Al Bairmany, Dr. George Chehadeh, Dr. Roy Jurdi, Dr. Shadi Saikaly, Dr. Ossama Abo Selo, Dr. Lara Hilal, Dr. Rana Salem, Mrs Dalya Salem Nehme. The authors would like to acknowledge the support for the satellite APCCC-MEPCC 2017 meeting in Beirut by Astellas, Janssen and Algorithm. Support for the 2019 workshop was provided by a nonrestricted educational grant by Astellas.

Author contributions DM: project development and manuscript writing/editing. BY: manuscript writing/editing. CD: manuscript writing/ editing. AEH: manuscript writing/editing. RN: manuscript writing/ editing. FG: manuscript writing/editing. DR: manuscript writing/editing. SAD: manuscript writing/editing. RS: manuscript writing/editing. RA: manuscript writing/editing. WW: manuscript writing/editing. MJ: manuscript writing/editing. GF: manuscript writing/editing. NAH: manuscript writing/editing. YAH: manuscript writing/editing. HG: manuscript writing/editing. HD: manuscript writing/editing. BB: manuscript writing/editing. CEK: manuscript writing/editing. GS: manuscript writing/editing. HKH: manuscript writing/editing. KS: manuscript writing/editing. KI: manuscript writing/editing. MH: manuscript writing/editing. NZ: manuscript writing/editing. NB: manuscript writing/editing. WA: manuscript writing/editing. FA: manuscript writing/editing. SF: manuscript writing/editing. EN: manuscript writing/ editing. GA: manuscript writing/editing. FF: manuscript writing/editing. MB: manuscript writing/editing. ST: manuscript writing/editing.
AS: manuscript writing/editing. SG: project development and manuscript writing/editing. AO: project development and manuscript writing/editing. RK: project development and manuscript writing/editing.

\section{Compliance with ethical standards}

Conflict of interest D Mukherji: research funding/honoraria from Astellas, Janssen, and Sanofi. A. Shamseddine: research funding/honoraria from Astellas, Janssen, and Sanofi. R. Khauli: research funding/ honoraria from Astellas, Janssen, Hickma, and Algorithm. S. Gillessen: advisory role (including IDMCs) and Speakers Bureau: AAA International, Active Biotech, Amgen, Astellas Pharma, Bayer, Bristol-Myers Squibb, CellSearch, Clovis, CureVac, Dendreon, ESSA Pharmaceuticals, Ferring, Innocrin Pharmaceuticals, Janssen Cilag, MaxiVAX SA, Millenium, Nectar, Novartis, Orion, Pfizer, ProteoMediX, Roche, and Sanofi. Co-inventor on patent application (WO 2009138392 A1) for a method for biomarker discover (granted in China, Europe, Japan and the US). Deputy of the ESMO guidelines committee for GU cancers, member of the EAU guideline panel for prostate cancer, past chair of the EORTC GU group. Member of the STAMPEDE trial management group. All the other authors have no relevant conflicts of interest to declare.

\section{Research involving human participants and/or animals None.}

Open Access This article is distributed under the terms of the Creative Commons Attribution 4.0 International License (http://creativeco mmons.org/licenses/by/4.0/), which permits unrestricted use, distribution, and reproduction in any medium, provided you give appropriate credit to the original author(s) and the source, provide a link to the Creative Commons license, and indicate if changes were made.

\section{References}

1. Gillessen S et al (2018) Management of patients with advanced prostate cancer: the report of the advanced prostate cancer consensus conference APCCC 2017. Eur Urol 73(2):178-211

2. Diamond IR et al (2014) Defining consensus: a systematic review recommends methodologic criteria for reporting of Delphi studies. J Clin Epidemiol 67(4):401-409

3. Al-Sukhun S et al (2018) ASCO resource-stratified guidelines: methods and opportunities. J Glob Oncol 4:1-8

4. Bray F et al (2018) Global cancer statistics 2018: GLOBOCAN estimates of incidence and mortality worldwide for 36 cancers in 185 countries. CA Cancer J Clin 68(6):394-424

5. Bazarbashi $\mathrm{S}$ et al (2014) Metastatic castration resistant prostate cancer: current strategies of management in the Middle East. Crit Rev Oncol Hematol 90(1):36-48 
6. Herget KA et al (2016) Recent decline in prostate cancer incidence in the United States, by age, stage, and Gleason score. Cancer Med $5(1): 136-141$

7. Mukherji D et al (2017) Prostate cancer stage at diagnosis: first data from a Middle-Eastern cohort. J Clin Oncol 35(6 suppl):e552-e552

8. Kasivisvanathan V et al (2018) MRI-targeted or standard biopsy for prostate-cancer diagnosis. N Engl J Med 378(19):1767-1777

9. El-Shater Bosaily A et al (2015) PROMIS-prostate MR imaging study: a paired validating cohort study evaluating the role of multi-parametric MRI in men with clinical suspicion of prostate cancer. Contemp Clin Trials 42:26-40

10. Parker CC et al (2018) Radiotherapy to the primary tumour for newly diagnosed, metastatic prostate cancer (STAMPEDE): a randomised controlled phase 3 trial. Lancet 392(10162):2353-2366

11. James ND et al (2017) Abiraterone for prostate cancer not previously treated with hormone therapy. N Engl J Med 377(4):338-351

12. James ND et al (2016) Addition of docetaxel, zoledronic acid, or both to first-line long-term hormone therapy in prostate cancer (STAMPEDE): survival results from an adaptive, multiarm, multistage, platform randomised controlled trial. Lancet 387(10024):1163-1177

13. Sweeney CJ et al (2015) Chemohormonal therapy in metastatic hormone-sensitive prostate cancer. N Engl J Med 373(8):737-746

14. Fizazi K et al (2017) Abiraterone plus prednisone in metastatic, castration-sensitive prostate cancer. N Engl J Med 377(4):352-360

15. Gandaglia $G$ et al (2014) Distribution of metastatic sites in patients with prostate cancer: a population-based analysis. Prostate $74(2): 210-216$

16. Viers BR et al (2014) Primary Gleason grade 4 at the positive margin is associated with metastasis and death among patients with Gleason 7 prostate cancer undergoing radical prostatectomy. Eur Urol 66(6):1116-1124

17. Carrie C et al (2016) Salvage radiotherapy with or without short-term hormone therapy for rising prostate-specific antigen concentration after radical prostatectomy (GETUG-AFU 16): a randomised, multicentre, open-label phase 3 trial. Lancet Oncol 17(6):747-756

18. Bianchi $\mathrm{L}$ et al (2016) The role of prostate-specific antigen persistence after radical prostatectomy for the prediction of clinical progression and cancer-specific mortality in node-positive prostate cancer patients. Eur Urol 69(6):1142-1148

19. Stephenson AJ et al (2007) Predicting the outcome of salvage radiation therapy for recurrent prostate cancer after radical prostatectomy. J Clin Oncol 25(15):2035-2041

20. Fossati $\mathrm{N}$ et al (2017) Impact of early salvage radiation therapy in patients with persistently elevated or rising prostate-specific antigen after radical prostatectomy. Eur Urol

21. Abugharib A et al (2017) Very early salvage radiotherapy improves distant metastasis-free survival. J Urol 197(3 Pt 1):662-668

22. Perera $\mathrm{M}$ et al (2019) Gallium-68 prostate-specific membrane antigen positron emission tomography in advanced prostate cancerupdated diagnostic utility, sensitivity, specificity, and distribution of prostate-specific membrane antigen-avid lesions: a systematic review and meta-analysis. Eur Urol

23. Shipley WU et al (2017) Radiation with or without antiandrogen therapy in recurrent prostate cancer. N Engl J Med 376(5):417-428

24. Rosenthal SA et al (2019) Effect of chemotherapy with docetaxel with androgen suppression and radiotherapy for localized highrisk prostate cancer: the randomized phase III NRG oncology RTOG 0521 trial. J Clin Oncol JCO1802158
25. Viani GA et al (2016) Intensity-modulated radiotherapy reduces toxicity with similar biochemical control compared with 3-dimensional conformal radiotherapy for prostate cancer: a randomized clinical trial. Cancer 122(13):2004-2011

26. Morgan SC et al (2018) Hypofractionated radiation therapy for localized prostate cancer: executive summary of an ASTRO, ASCO, and AUA evidence-based guideline. Pract Radiat Oncol $8(6): 354-360$

27. Nabid A et al (2018) Duration of androgen deprivation therapy in high-risk prostate cancer: a randomized phase III trial. Eur Urol 74(4):432-441

28. Geara FB et al (2017) Nadir PSA is a strong predictor of treatment outcome in intermediate and high risk localized prostate cancer patients treated by definitive external beam radiotherapy and androgen deprivation. Radiat Oncol 12(1):149

29. Smith MR, Yu MK, Small EJ (2018) Apalutamide and metastasisfree survival in prostate cancer. N Engl J Med 378(26):2542

30. Hussain $\mathrm{M}$ et al (2018) Enzalutamide in men with nonmetastatic, castration-resistant prostate cancer. $\mathrm{N}$ Engl J Med 378(26):2465-2474

31. Fizazi K et al (2019) Darolutamide in nonmetastatic, castrationresistant prostate cancer. N Engl J Med 380(13):1235-1246

32. Sydes MR et al (2018) Adding abiraterone or docetaxel to longterm hormone therapy for prostate cancer: directly randomised data from the STAMPEDE multi-arm, multi-stage platform protocol. Ann Oncol 29(5):1235-1248

33. Aguiar PN, Jr et al (2019) Cost-effectiveness analysis of abiraterone, docetaxel or placebo plus androgen deprivation therapy for hormone-sensitive advanced prostate cancer. Einstein (Sao Paulo) 17(2):eGS4414

34. Kyriakopoulos CE et al (2018) Chemohormonal therapy in metastatic hormone-sensitive prostate cancer: long-term survival analysis of the randomized phase III E3805 CHAARTED trial. J Clin Oncol 36(11):1080-1087

35. Szmulewitz RZ et al (2018) Prospective international randomized phase II study of low-dose abiraterone with food versus standard dose abiraterone in castration-resistant prostate cancer. J Clin Oncol 36(14):1389-1395

36. Aggarwal R et al (2018) Clinical and genomic characterization of treatment-emergent small-cell neuroendocrine prostate cancer: a multi-institutional prospective study. J Clin Oncol 36(24):2492-2503

37. Chakhtoura M et al (2017) The FRAX-based Lebanese osteoporosis treatment guidelines: rationale for a hybrid model. Osteoporos Int 28(1):127-137

38. Fizazi K et al (2011) Denosumab versus zoledronic acid for treatment of bone metastases in men with castration-resistant prostate cancer: a randomised, double-blind study. Lancet 377(9768):813-822

39. Saad F (2002) Zoledronic acid significantly reduces pathologic fractures in patients with advanced-stage prostate cancer metastatic to bone. Clin Prostate Cancer 1(3):145-152

40. Saad F et al (2004) Long-term efficacy of zoledronic acid for the prevention of skeletal complications in patients with metastatic hormone-refractory prostate cancer. J Natl Cancer Inst 96(11):879-882

41. Himelstein AL et al (2017) Effect of longer-interval vs standard dosing of zoledronic acid on skeletal events in patients with bone metastases: a randomized clinical trial. JAMA 317(1):48-58

Publisher's Note Springer Nature remains neutral with regard to jurisdictional claims in published maps and institutional affiliations. 


\section{Affiliations}

Deborah Mukherji $^{1}$ (I) Bassem Youssef ${ }^{2} \cdot$ Christelle Dagher $^{1} \cdot$ Albert El-Hajj $^{3} \cdot$ Rami Nasr $^{3} \cdot$ Fadi Geara $^{2}$. Danny Rabah ${ }^{4}$ - Saad AI Dousari ${ }^{5} \cdot$ Rabih Said $^{6} \cdot$ Raja Ashou $^{7}$ - Wassim Wazzan ${ }^{2}$. Michel Jabbour ${ }^{8}$. George Farha ${ }^{9}$. Nibras Al Hamdani ${ }^{10}$. Yousuf Al Hallaq ${ }^{10}$. Hassan Ghazal ${ }^{11}$. Haifa Dbouk ${ }^{12}$. Bassel Bachir ${ }^{2}$. Clement El Khoury ${ }^{13}$. Ghazi Sakr $^{14}$. Hero K. Hussain ${ }^{15} \cdot$ Khaled Sayyid $^{16} \cdot$ Khaled Ibrahim $^{17} \cdot$ Mohammad Haidar $^{15} \cdot$ Nicolas Zouain $^{18}$. Nizar Bitar ${ }^{19}$. Walid Alameh ${ }^{20}$. Fadi Abbas ${ }^{21}$. Sami Faddoul ${ }^{22}$. Elie Nemer ${ }^{23}$. Georges Assaf ${ }^{8}$. Fadi Farhat ${ }^{17}$. Muhammad Bulbul $^{2} \cdot$ Sally Temraz ${ }^{1}$. Ali Shamseddine ${ }^{1} \cdot$ Silke Gillessen $^{24,25} \cdot$ Aurelius Omlin $^{25} \cdot$ Raja Khauli $^{3}$

1 Division of Hematology/Oncology, Department of Internal Medicine, American University of Beirut Medical Center, Riad El Solh, 11072020 Beirut, Lebanon

2 Department of Radiation Oncology, American University of Beirut Medical Center, Beirut, Lebanon

3 Division of Urology, Department of Surgery, American University of Beirut Medical Center, Riad El Solh, 11072020 Beirut, Lebanon

4 Department of Surgery, College of Medicine, King Saud University, Riyadh, Saudi Arabia

5 Department of Surgery, Faculty of Medicine, Kuwait University, Kuwait City, Kuwait

6 Division of Hematology/Oncology, Department of Internal Medicine, Saint Georges Hospital University Medical Center, Beirut, Lebanon

7 Department of Radiology, Saint Georges Hospital University Medical Center, Beirut, Lebanon

8 Division of Urology, Department of Surgery, Saint Georges Hospital University Medical Center, Beirut, Lebanon

9 Department of Radiation Oncology, Mount Lebanon Hospital, Faculty of Medicine-University of Balamand, Beirut, Lebanon

10 Department of Surgery, Medical City Teaching Hospital Baghdad, Baghdad, Iraq

11 Division of Hematology/Oncology, Department of Internal Medicine, Clemenceau Medical Center, Beirut, Lebanon

12 Division of Oncology, Jamal Amel Hospital, Tyre, Lebanon
13 Department of Radiation Oncology, Hotel Dieu de France, Saint Joseph University Faculty of Medicine, Beirut, Lebanon

14 Division of Urology, Department of Surgery, Mount Lebanon Hospital, Beirut, Lebanon

15 Department of Radiology, American University of Beirut Medical Center, Beirut, Lebanon

16 Department of Surgery, Makassed Hospital, Beirut, Lebanon

17 Division of Hematology/Oncology, Department of Internal Medicine, Hammoud Hospital University Medical Center, Saida, Lebanon

18 Department of Radiation Oncology, Clemenceau Medical Center, Beirut, Lebanon

19 Division of Hematology/Oncology, Department of Internal Medicine, Sahel General Hospital, Beirut, Lebanon

20 Department of Surgery, Sahel General Hospital, Beirut, Lebanon

21 Middle East Tumor Institute, Beirut, Lebanon

22 Doctors Center Radiology, Beirut, Lebanon

23 Department of Urology, Saint Joseph University, Beirut, Lebanon

24 Division of Cancer Sciences, University of Manchester and the Christie, Manchester, UK

25 Department of Medical Oncology and Haematology, Cantonal Hospital Saint Gallen, University of Bern, Bern, Switzerland 